\title{
MedienPädagogik
}

Zeitschrift für Theorie und Praxis der Medienbildung

\section{Editorial: Medienpädagogik in Zeiten einer tiefgreifenden Mediatisierung}

Karsten D. Wolf, Klaus Rummler, Patrick Bettinger und Sandra Aßmann

\section{Mediatisierung als eine Bezugstheorie der Medienpädagogik}

Mit dem Begriff der Digitalisierung ist die Beschäftigung mit Medien seit Mitte der 2010er Jahre (wieder) ins Zentrum der allgemeinen bildungspolitischen Diskussion gerückt. So hat in Deutschland das KMK-Strategiepapier «Bildung in der digitalen Welt» (2016) medienpädagogische Kompetenzen in den allgemeinen Pflichtkanon der Aus- und Weiterbildung von Lehrpersonen gehoben, während in der Schweiz der Themenkomplex «Medien und Informatik» als Teil des «Lehrplan 21» (Deutschschweizer Erziehungsdirektoren-Konferenz - D-EDK 2014) vollständig überarbeitet wurde und in Österreich das BMBWF die Digitalisierungsstrategie «Schule 4.0» (Bundesministerium für Bildung, Wissenschaft und Forschung 2017) implementiert. Auch in der ausserschulischen und der beruflichen Bildung gibt es einen breiten Konsens über die Notwendigkeit, sich intensiver mit den pädagogischen und didaktischen Konsequenzen eines Veränderungsprozesses zu beschäftigen.

Die Kommunikations- und Medienwissenschaft liefert mit dem Begriff der Mediatisierung den theoretischen Rahmen für die Beschreibung und der Frage nach dem Wechselbezug zwischen einem sich beschleunigenden Medienwandel, sowohl beharrenden als auch innovativen 
Kommunikationspraxen sowie einem globalen soziokulturellen Wandel (Krotz 2007). In der Mediatisierungstheorie geht es dabei gerade nicht um den Vergleich spezieller Medienformate wie z. B. Kreidetafel vs. Interactive Whiteboard oder deren spezifischen technologischen Verfasstheiten wie z. B. analog vs. digital. Im Mittelpunkt steht die Analyse der in der menschlichen Kulturgeschichte zu beobachtenden Veränderungen der kommunikativen Handlungspraxen, der Mediensysteme und der sich darauf konstruierenden sozialen Handlungskontexte sowie ihre wechselseitige Verschränkung (vgl. Hepp 2013). Dabei gibt es durchaus Parallelen in der Entwicklung der Medienpädagogik und Teilen der Kommunikations- und Medienwissenschaften. In Beiden hat sich eine Wandlung von der Betrachtung des Paares Massenmedien und rezipierendes Publikum hin zu Social Media und aktiver Partizipation der Benutzenden vollzogen (Krotz 2013). So wie die Medienpädagogik rein bewahrpädagogische Positionen überwunden hat und stattdessen besonders im Kontext der Medienkompetenzförderung von produktiv realitätsverarbeitenden Subjekten ausgeht, so fand in den Kommunikations- und Medienwissenschaften ebenfalls eine theoretische Entwicklung von einfachen Stimulus-Response-Ansätzen über Uses-and-Gratification-Modellen hin zum kontextuellen Verstehen der Medienaneignung statt.

Mediatisierung im Verständnis von Hepp und dem «Communicative Figurations〉 research network» (2017) beschreibt die wechselseitige Beeinflussung von Medien und Kommunikationsprozessen auf unterschiedlichen Ebenen. Aus dieser Perspektive wird Kultur, Gesellschaft und Individuen als interdependenter Zusammenhang betrachtet, welcher sich durch Mediatisierungsschübe - gegenwärtig in Form der Digitalität - verändert. Dabei beeinflussen Medien unsere kommunikativen Praktiken, Haltungen und Einstellungen in zunehmendem Masse auf einer Makroebene, während die individuelle Aneignung von Medien durch Menschen auf deren Gestaltung und Weiterentwicklung einwirken (Mikroebene). Die Mediatisierungstheorie beschreibt also auf einer Mesoebene die wechselseitige Beinflussung von Medien, Kultur und Gesellschaft: Medien sind «überall» und durchdringen alle sozialen Sphären wie z. B. die Politik, die Religion, aber auch die Bildung. 
Medien sind dabei nicht in isolierter Hinsicht als Agenten des Wandels zu verstehen, sondern als ein Element unter anderen, welches empirisch berücksichtigt und in Theorien integriert werden muss, um kommunikative - und damit alle sozialen - Prozesse zu analysieren, zu verstehen und zu modellieren. Gleichzeitig sind Medien aber so tief in unsere Gesellschaft und Kultur integriert, dass ein analytisches Herauslösen nicht mehr möglich erscheint (Hepp 2020).

Die Mediatisierungstheorie trägt durch diese Prämissen zu einem besseren Verständnis menschlicher Kommunikation (bzw. deren Wandel) bei und erweitert die Vorstellung der sozialen Konstruktion von Gesellschaft und Individuum vor dem Hintergrund gegenwärtiger medientechnologischer Entwicklungen. Demnach sind Bildung und Erziehung ohne einen solchen Einbezug medienkommunikativer Veränderungen kaum mehr zu verstehen oder zu gestalten. Auch methodisch stellt die mit der Mediatisierungstheorie verbundene empirische Erforschung des Medienhandelns einen wichtigen Beitrag zur Weiterentwicklung der handlungsorientierten Medienpädagogik dar, indem sie Wege zeigen, die komplexen sozialen, kulturellen und medialen Verflechtungen zu erschliessen.

\section{Tiefgreifende Mediatisierung als radikalisierte Version des Mediatisierungsprozesses}

Nicht erst seit der Corona-Pandemie ist die zunehmende Durchdringung aller Lebensräume durch digitale, überwiegend vernetzte und zunehmend mobile Medien Alltagspraxis. Standardisierte und regelhaft durchgeführte Untersuchungen wie die ARD/ZDF-Onlinestudie bzw. die KIM- und JIM-Studien zeichnen eindrücklich die veränderten Nutzungsmuster über die letzten zwei Jahrzehnte nach, z. B. über erste Social Network Sites wie myspace über SchülerVZ und Facebook hin zu WhatsApp, Instagram und Snapchat. Ähnlich wie bei der sich verändernden Nutzung klassischer Medien wie Radio und Fernsehen unterliegt die Social-Media-Mediennutzung einem Wandel. So diente z. B. YouTube sehr lange den Jugendlichen nicht nur als «zentrale Anlaufstelle für Bewegtbildnutzung» (Busemann und Gscheidle 2012, 388 f.), sondern auch als kostenlose Musikquelle, bis 
Musik-Streamingdienste wie Spotify diese Rolle diese teilweise übernahmen. An diesem Beispiel wird deutlich, dass die Nutzungsveränderung von Medien nicht die Ausnahme darstellt, sondern vielmehr die Regel.

Obwohl es historisch betrachtet bereits in der Vergangenheit immer wieder zu Mediatisierungsschüben kam, postuliert das «Communicative Figurations〉 Research Network» an den Universitäten Bremen und Hamburg mit Blick auf aktuelle Entwicklungen einen Unterschied, der unter anderem darin besteht, dass sich der Mediatisierungsprozess in der letzten Dekade intensiviert hat (Hepp und «Communicative Figurations» research network 2017; Couldry und Hepp 2016). Diese aktuelle Phase einer radikalisierten Form der Mediatisierung wird als tiefgreifende Mediatisierung bezeichnet: Die soziale Bedeutung von Mediatisierung ist nicht mehr nur auf spezifische Subsysteme der Gesellschaft, z. B. den Journalismus zu reduzieren. Alle Teilbereiche sind - wenn auch unterschiedlich - davon betroffen. Insbesondere in der Medienpädagogik ist herauszuarbeiten, auf welche Weise und in welchem Masse der Bildungsbereich von der zunehmenden Digitalisierung der technischen Kommunikationsmedien betroffen ist und welche pädagogischen Konsequenzen daraus zu ziehen sind.

Der Prozess einer tiefgreifenden Mediatisierung vollzieht sich weder gleichförmig, linear oder gleichgerichtet über alle gesellschaftlichen Subsysteme. So entzog sich die Unterrichtspraxis in Schulen vor der CoronaPandemie lange einer fortschreitenden Digitalisierung. Ein «Ignorieren» oder «Ablehnen» dieses allumfassenden Veränderungsdispositivs ist nur punktuell möglich, langfristige Vermeidungsstrategien können sich dagegen sogar als dysfunktional erweisen: so sind z. B. das Verhandeln von Zeiträumen für die Nicht-Nutzung von Smartphones in Schulen oder in der Familie lediglich temporäre Phasen eines schulischen bzw. familiären «digitalen Detox» (Wolf 2017, 339) und es wird immer deutlicher, dass stattdessen eine produktive und pädagogisch sinnvolle Anwendung von ohnehin im Alltag der Kinder und Jugendlichen fest verankerten Mediennutzungspraktiken zielführender ist.

Diese fortgeschrittene Phase einer tiefgreifenden Mediatisierung wird durch zumindest fünf Trends gekennzeichnet, welche den aktuellen Wandel in besonderer Weise bestimmen (vgl. Hepp 2018). Inwieweit diese Trends dauerhaft Bestand haben (werden), oder welche anderen Trends 
sich entwickeln werden, ist zum heutigen Zeitpunkt nicht vorherzusagen. Allerdings helfen sie, die qualitative Veränderung der mediatisierten Lebenswelten in einer digitalen Gesellschaft zu verstehen und zu analysieren (Hepp und «Communicative Figurations» research network 2017):

- eine grösser werdende Differenzierung der Medienformen, -formate und -typen

- eine zunehmende Konnektivität bzw. Vernetzung der Medien bzw. durch Medien, welche Raum und Zeit überbrücken

- eine wachsende Omnipräsenz der Medien, auf die zu jeder Zeit und von überall zugegriffen werden kann

- eine sich verstärkende Innovationsgeschwindigkeit mit der neue Medien und Kommunikationsdienste eingeführt werden

- eine intensivierende Datafizierung, also die digitale Verarbeitung und Speicherung von Daten durch Geräte wie z. B. Smartphones oder Wearables, deren Software sowie in der zugrundeliegenden digitalen Infrastruktur.

\section{Beiträge in diesem Band}

Das Ziel dieses 16. Jahrbuch Medienpädagogik ist es, die vielschichtigen und beschleunigten Wandlungsprozesse im Sinne einer tiefgreifenden Mediatisierung aus der Perspektive der Medienpädagogik und den mediendidaktisch-orientierten Fachdidaktiken in ihrer Bedeutung für die Themenfelder Bildung, Lehren, Lernen, Erziehung und Sozialisation zu interpretieren, zu erforschen und zu gestalten. Die Beiträge wurden auf der Herbsttagung der Sektion Medienpädagogik der Deutschen Gesellschaft für Erziehungswissenschaft (DGfE) am 20. und 21. September 2018 an der Universität Bremen als Posterbeiträge oder Präsentationen vorgestellt und diskutiert.

Andreas Dertinger (S. 1-27) erörtert die Frage, wie das Konstrukt des Habitus mit den gesellschaftlichen Veränderungen einer tiefgreifenden Mediatisierung in Beziehung gesetzt werden kann. Er geht dabei von der These aus, dass die tiefgreifende Mediatisierung zu einer essenziellen Veränderung der Strukturbedingungen des pädagogischen Medienhandelns in der 
Schule führt. Obwohl es nicht an Beschreibungen für die sich daraus ergebenden medienpädagogischen Kompetenzen von Lehrkräften mangelt, ist eine erfolgreiche digitale Professionalierung keineswegs gesichert. Als eine mögliche Ursache dafür arbeitet Dertinger aus der Perspektive der praxeologischen Wissenssoziologie das Spannungsfeld zwischen (Kompetenz-)Erwartungen und dem jeweils persönlichem Habitus der Lehrkräfte heraus. Das Habitus-Konstrukt impliziert dabei nicht automatisch ein Scheitern, sondern kann bei einem Teil der Lehrkräfte auch ein Gelingen erklären. Indem der Ansatz eines habituell beeinflussten professionellen Medienhandelns von Lehrpersonen mit struktur- und kompetenztheoretischen Professionsansätzen in Verbindung gesetzt wird, soll ein besseres theoretisches Verständnis für die Weiterentwicklung von Professionalisierungansätze gelegt werden.

Henriette Mehlan und Jörg Holten (S. 29-49) greifen den Begriff der Medienkompetenz vor dem Kontext der Mediatisierungstheorie erneut auf. Dazu unterscheiden sie definitorisch zunächst zwischen einem nominalen, operationalen und normativen Begriffsverständnis von (Medien)Kompetenz. Im Anschluss an Anthony Giddens werden in einem nächsten Schritt Kompetenz und Performanz als zwei Dimension unterschieden (Dualität), die sich gegenseitig bedingen und reproduzieren. Durch eine weitere Unterscheidung zwischen Wirklichkeitskonstruktionen erster und zweiter Ordnung machen Mehlan und Holten bisher implizite Denkweisen über Kompetenz sichtbar. Ihre daraus abgeleitete Kompetenzdefinition bezieht sich folglich nicht auf eine reine Aufschlüsselung einzelner Kompetenzen, sondern impliziert die Frage nach der Dualität von Kompetenz in einer tiefgreifend mediatisierten Welt.

Ilka Koppel (S. 51-78) stellt aus der Perspektive der Erwachsenenbildung die Frage, welche Faktoren zu einem gelingenden Einsatz digitaler Medien in der Alphabetisierung und Grundbildung beitragen können. Ausgehend von einer Analyse der sich aus einer tiefgreifenden Mediatisierung ergebenden veränderten Anforderungen und Rahmenbedingungen einer Grundbildung wird im Beitrag auf den möglichen Einsatz digitaler Medien in Angeboten der Grundbildung, z. B. an Volkshochschulen, fokussiert. Die Ergebnisse der qualitativen Analyse einer Interviewstudie mit 8 Expertinnen 
und Experten stellt sie auf individueller, institutioneller und prozessualer Ebene dar und diskutiert sie. Schlussfolgernd werden Gelingensbedingungen für einen erfolgreichen Einsatz digitaler Medien im Alphabetisierungs- und Grundbildungsbereich auf den drei Ebenen vorgestellt sowie die Studie reflektiert und weitere Forschungsdesiderata entwickelt.

In ihrer Forschungsarbeit analysiert Caroline Grabensteiner (S. 79-107) im Rahmen einer Mixed-Methods-Studie die Rolle von Kommunikationsplattformen bei der Organisation der Lernaktivitäten von Schülerinnen und Schüler bezogen auf mediale Relationen, Agency und Bildungsrelationen. Schulklassen werden als kommunikative Figurationen modelliert, um die entsprechenden Akteurskonstellationen und Medienrepertoires zu identifizieren. Dazu werden Ansätze der Sozialen Netzwerkanalyse (SNA) mit interpretativen Methoden der Datenauswertung kombiniert. Durch diese Methodenkombination gelingt es, das Zusammenspiel zwischen individuellem und kollektivem Medienhandeln in Bezug auf Lernaktivitäten zu rekonstruieren.

In ihrer empirischen Studie untersuchen Sebastian Wachs und Michelle E. Wright (S. 109-128) die bisher kaum erforschte Überlappung zwischen Viktimisierung und Täterschaft bei Online-Hate, um ein besseres Verständnis der Beziehung von Online-Hate-Opfern und -Täterinnen oder -Tätern zu entwickeln sowie, um Grundlagen für wirksame Interventionsund Präventionsinitiativen im Bereich der Medienerziehung zu schaffen. Auf Basis einer Fragebogenstudie mit 1.480 Jugendlichen zwischen 12 und 17 Jahren arbeiten sie heraus, dass effektive Präventions- und Interventionsprogramme in Bezug auf Online-Hate insbesondere problemfokussierte Bewältigungsstrategien vermitteln sollten, um die Wahrscheinlichkeit revanchierender Online-Hate durch Opfer zu verringern.

Lara Gerhardts, Lukas Dehmel und Dorothee E. Meister (S. 129-159) nähern sich einer zentralen Fragestellung zu Gelingensbedingungen digitaler Ausstattungsinitiativen von Schulen auf methodisch innovative Weise. Der berufliche Aneignungsprozess von Diensttablets durch Lehrkräfte wird als Ausbildung eines «Hybrid-Akteurs» basierend auf der Theorie einer «Pädagogik der Dinge» nach Arnd-Michael Nohl interpretiert 
und empirisch untersucht. Dazu werden besonders aneignungsrelevante Faktoren der Überwindung technikinduzierter Irritationen identifiziert. Neben der allgemeinen Grundhaltung zum schulischen Einsatz digitaler Medien und der bereits vorhandenen Medienkompetenz der Lehrenden ist die Unterstützung der Lehrkraft währen des Aneignungsprozesses von besonderer Bedeutung. Auf Basis der Ergebnisse werden Optimierungspotenziale für Einführungs- und Unterstützungsprogramme diskutiert.

Stefanie Nickel (S. 161-184) beschreibt und analysiert ein Projekt aus der Lehramtsausbildung, um kooperative und kommunikative Erfahrungsräume beim Einsatz digitaler Medien zu schaffen. Im Fokus steht dabei, neben der Anbahnung und dem Aufbau von Medienkompetenz durch den Umgang mit digitalen Medien, ein dialogisch orientierter Aushandlungsprozess zwischen den Studierenden und den Schülerinnen und Schüler in Kleingruppen. Dabei sollen hierarchiefrei heterogene Medienerfahrungen eingebracht werden können, damit sich die Lehramtsstudierenden das Lebensumfeld der Schülerinnen und Schüler erschliessen können und nicht nur ihre eigenen Überzeugungen vermitteln. Dazu erkunden und erschliessen sich die beteiligten Akteurinnen und Akteure gemeinsam im Team einen bestimmten (ausserschulischen) Ort, um im Anschluss eine Rallye zu planen, auszuarbeiten sowie zu reflektieren.

Rudolf Kammerl, Claudia Lampert, Jane Müller, Marcel Rechlitz und Katrin Potzel (S. 185-209) erörtern die methodologischen Anforderungen, welche eine zunehmende Mediatisierung kindlicher Lebenswelten für die Erforschung mediatisierter Sozialisationsprozesse darstellen. Es wird ein Mangel an Studien zur Rolle der sich ändernden Medienumgebung für die Sozialisation identifiziert. Insbesondere gibt es kaum Projekte, welche eine umfassende Sicht wie z. B. das Zusammenspiel verschiedener Medientypen in den Blick nehmen. Als eine passende theoretische Rahmung wird das Konzept der kommunikativen Figurationen anhand seiner konstituierenden Merkmale vorgestellt. Es wird begründet, warum das Figurationskonzept besonders gut geeignet ist, Sozialisationsprozesse in einer Kultur der tiefgreifenden Mediatisierung zu erforschen und warum dies idealerweise im Längsschnitt geschehen muss. 
Nina Grünberger (S. 211-229) versucht einen möglichen methodologischen Zugang zu einer weltumspannenden Medienpädagogik zu skizzieren, welche ökologische Implikationen der Ubiquität digitaler Technologien mit in einen kritischen Diskurs eines ökologisch-nachhaltigen Medienhandelns einbindet. Dazu werden in einem interdisziplinären Zugang neben medienpädagogischen und medienwissenschaftlichen Überlegungen auch Positionen einer «Bildung für nachhaltige Entwicklung» sowie Postcolonial Studies einbezogen, um die Dominanz des Globalen Nordens in den Diskursen zu Digitalisierung, Mediatisierung, Medialisierung sowie Digitalität aufzubrechen. Aus dieser Analyse ergeben sich wichtige neue medienpädagogische Anschlussfelder sowie Forschungsfragen, welche abschliessend vorgestellt und diskutiert werden.

Dieter Spanhel (S. 231-280) vertritt die These, dass unter den Bedingungen mediatisierter Lebenswelten ein «gedeihliches Aufwachsen mit Medien» nur durch aufeinander abgestimmte Massnahmen des Kinder- und Jugendmedienschutzes sowie der Medienpädagogik gesichert werden können. Zur Begründung dieser These analysiert Dieter Spanhel die mediatisierten Kontexte Familie, Kita, Schule und Freizeit aus einer systemtheoretischen Perspektive, um die Faktoren aufzudecken, welche auf das selbstregulierte Medienhandeln der Heranwachsenden einwirken. Sowohl dem Medienschutz als auch medienpädagogischen Massnahmen werden dabei notwendige, aber gleichwohl begrenzte Einflussmöglichkeiten zugesprochen. Aus dieser Problematik heraus entwickelt Spanhel das Konzept einer kontextuellen Steuerung des Medienhandelns der Heranwachsenden als ein Modell medienpädagogischen Handelns, welches Schutzfunktion und pädagogische Unterstützung verbindet und sich an den Sinnkriterien intellektueller und moralischer Autonomie und der Befähigung zu verantwortlicher sozialer Teilhabe ausrichtet. Für dessen praktische Umsetzung werden Prinzipien, Ansatzpunkte und konkrete Beispiele aufgezeigt.

Lukas Gerthofer und Jennifer Schneider (S. 281-315) untersuchen die Forschungsfrage, welche Konstellationen von personenbezogenen Ursachen von Lehrpersonen zu (mangelndem) Einsatz digitaler Medien im Unterricht führen. Auf Basis des aus der Technologieakzeptanzforschung 
stammenden Modells der «Unified theory of acceptance and use of technology» (UTAUT) wird ein qualitatives Forschungsdesign mit einem fallstudienähnlichen und explanativen Charakter konzipiert und im Rahmen von Expertinnen- und Experten-Interviews mit 12 Lehrkräften umgesetzt. In der qualitativen Auswertung kann zunächst das UTAUT-Modell global weitgehend bestätigt werden, allerdings zeigen sich in einer Einzelfallanalyse der interviewten Personen, dass nicht alle Faktoren für die tatsächliche Nutzung relevant sind und teilweise auch nicht vollständig im Modell abgebildet werden. Abschliessend werden Hinweise zu besonders förderlichen Massnahmen zur Unterstützung der Einführung neuer Technologien im Schulkontext gegeben.

\section{Ingrid Darmann-Finck, Claudia Schepers, Karsten D. Wolf und Jan Küster} (S. 317-345) verschränken fachdidaktische Anforderungen aus der Pflegedidaktik mit mediendidaktischen Gestaltungsprinzipien für die Entwicklung digitaler Unterrichtsinhalte innerhalb eines Classroom-LearningInteraction-Systems (CLIS). Auf Basis pflegedidaktischer Grundlagen wird dazu ein mediendidaktisches Design konzipiert. Sie zeigen dabei die iterative Entwicklung sowohl der Software (CARO CLIS) als auch der Unterrichtsinhalte auf Basis des Design-based Research Ansatzes auf. Exemplarisch wird dabei dargestellt, wie mit der Software pflegedidaktisch fundierte komplexe Bildungsziele mit Hilfe mediendidaktischer Interaktionsformate im Unterricht umgesetzt werden können.

\section{Literatur}

Bundesministerium für Bildung, Wissenschaft und Forschung. 2017. «Schule 4.0. - jetzt wird's digital». 2017. https://bildung.bmbwf.gv.at/schulen/schule40/ index.html.

Busemann, Katrin, und Christoph Gscheidle. 2012. «Web 2.0: Habitualisierung der Social Communitys. Ergebnisse der ARD/ZDF-Onlinestudie 2012». Media Perspektiven 7-8: 380-90. https://www.ard-zdf-onlinestudie.de/ files/2012/0708-2012_Busemann_Gscheidle.pdf.

Couldry, Nick, und Andreas Hepp. 2016. The mediated construction of reality. Cambridge: Polity Press.

Deutschschweizer Erziehungsdirektoren-Konferenz - D-EDK, Hrsg. 2014. «Lehrplan 21: Medien und Informatik». D-EDK. http://v-ef.lehrplan.ch/container/V_ EF_DE_Modul_MI.pdf. 
Hepp, Andreas. 2013. Medienkultur. Die Kultur mediatisierter Welten. Medien - Kultur - Kommunikation. Wiesbaden: VS Verlag für Sozialwissenschaften. https://doi.org/10.1007/978-3-531-19933-7.

Hepp, Andreas. 2018. «Von der Mediatisierung zur tiefgreifenden Mediatisierung». In Kommunikation - Medien - Konstruktion. Braucht die Mediatisierungsforschung den Kommunikativen Konstruktivismus?, herausgegeben von Jo Reichertz und Richard Bettmann, 27-45. Wissen, Kommunikation und Gesellschaft. Schriften zur Wissenssoziologie. Wiesbaden: Springer Fachmedien Wiesbaden. https://doi.org/10.1007/978-3-658-21204-9_2.

Hepp, Andreas. 2020. Deep Mediatization. Key Ideas in Media \& Cultural Studies. London; New York: Routledge. https://doi.org/10.4324/9781351064903.

Hepp, Andreas und «Communicative Figurations» research network. 2017. «Transforming Communications - Media -related Changes in Times of Deep Mediatization». Communicative Figurations Working Paper Series, Working Paper, Nr. 16. http://nbn-resolving.de/urn:nbn:de:gbv:46-00105985-11.

KMK - Kultusministerkonferenz. 2016. «Bildung in der digitalen Welt. Strategie der Kultusministerkonferenz. Beschluss der Kultusministerkonferenz vom 08.12.2016 in der Fassung vom 07.12.2017». Kultusministerkonferenz. https:// www.kmk.org/fileadmin/Dateien/pdf/PresseUndAktuelles/2018/Digitalstrategie_2017_mit_Weiterbildung.pdf.

Krotz, Friedrich. 2007. Mediatisierung. Fallstudien zum Wandel von Kommunikation. Medien, Kultur, Kommunikation. Wiesbaden: VS Verlag für Sozialwissenschaften. https://doi.org/10.1007/978-3-531-90414-6.

Krotz, Friedrich. 2013. "Aufwachsen in mediatisierten Welten». In Medienwelten im Wandel: Kommunikationswissenschaftliche Positionen, Perspektiven und Konsequenzen, herausgegeben von Christine W. Wijnen, Sascha Trültzsch, und Christina Ortner, 39-53. Wiesbaden: Springer Fachmedien. https://doi. org/10.1007/978-3-531-19049-5_4.

Wolf, Karsten D. 2017. «Mediatisierung in reformpädagogischen Lernkulturen». In Handbuch Reformpädagogik, herausgegeben von Till-Sebastian Idel und Heiner Ullrich, 338-52. Beltz-Handbuch. Weinheim: Beltz. 


\section{Beiträge}

Darmann-Finck, Ingrid, Karsten D. Wolf, Claudia Schepers, und Jan Küster. 2021. «Digital unterstütztes Lernen in der Pflegeausbildung: Die Care Reflection Online (CARO) - Lernumgebung». Herausgegeben von Karsten D. Wolf, Klaus Rummler, Patrick Bettinger, und Sandra Aßmann. MedienPädagogik: Zeitschrift für Theorie und Praxis der Medienbildung, Jahrbuch Medienpädagogik, 16 (Jahrbuch Medienpädagogik): 317-45. https://doi.org/10.21240/mpaed/ jb16/2021.04.30.X.

Dertinger, Andreas. 2021. «Medienpädagogische Professionalisierung von Lehrpersonen in einer mediatisierten Welt: Der Habitus als Bindeglied zwischen gesellschaftlichen Anforderungen und pädagogischem Medienhandeln». Herausgegeben von Karsten D. Wolf, Klaus Rummler, Patrick Bettinger, und Sandra Aßmann. MedienPädagogik: Zeitschrift für Theorie und Praxis der Medienbildung, Jahrbuch Medienpädagogik, 16 (Jahrbuch Medienpädagogik): 1-27. https://doi.org/10.21240/mpaed/jb16/2021.01.10.X.

Gerhardts, Lara, Lukas Dehmel, und Dorothee M. Meister. 2021. «Die berufliche Tabletaneignung von Lehrkräften als Beispiel der Mediatisierung pädagogischer Handlungskontexte: Theoretische Perspektiven und empirische Befunde». Herausgegeben von Karsten D. Wolf, Klaus Rummler, Patrick Bettinger, und Sandra Aßmann. MedienPädagogik: Zeitschrift für Theorie und Praxis der Medienbildung, Jahrbuch Medienpädagogik, 16 (Jahrbuch Medienpädagogik): 129-59. https://doi.org/10.21240/mpaed/jb16/2021.01.15.X.

Gerthofer, Lukas, und Jennifer Schneider. 2021. «Fallkonstellationen zum Einsatz digitaler Medien im Unterricht: Eine qualitative, lehrendenzentrierte Betrachtung». Herausgegeben von Karsten D. Wolf, Klaus Rummler, Patrick Bettinger, und Sandra Aßmann. MedienPädagogik: Zeitschrift für Theorie und Praxis der Medienbildung, Jahrbuch Medienpädagogik, 16 (Jahrbuch Medienpädagogik): 281-315. https://doi.org/10.21240/mpaed/jb16/2021.04.29.X.

Grabensteiner, Caroline. 2021. «Die vernetzte Schulklasse: Exploration zu Konstruktionen individueller und kollektiver Lernaktivitäten am Beispiel von WhatsApp-Gruppenchats». Herausgegeben von Karsten D. Wolf, Klaus Rummler, Patrick Bettinger, und Sandra Aßmann. MedienPädagogik: Zeitschrift für Theorie und Praxis der Medienbildung, Jahrbuch Medienpädagogik, 16 (Jahrbuch Medienpädagogik): 79-107. https://doi.org/10.21240/mpaed/ jb16/2021.01.13.X.

Grünberger, Nina. 2021. «Postkolonial post-digital: Forschungsfelder und Anschlussstellen für die Medienpädagogik durch eine postkoloniale Perspektive auf eine Post-Digitalität». Herausgegeben von Karsten D. Wolf, Klaus Rummler, Patrick Bettinger, und Sandra Aßmann. MedienPädagogik: Zeitschrift für Theorie und Praxis der Medienbildung, Jahrbuch Medienpädagogik, 16 (Jahrbuch Medienpädagogik): 211-29. https://doi.org/10.21240/mpaed/jb16/2021.02.25. 
Kammerl, Rudolf, Claudia Lampert, Jane Müller, Marcel Rechlitz, und Katrin Potzel. 2021. «Mediatisierte Sozialisationsprozesse erforschen: Methodologische Implikationen». Herausgegeben von Karsten D. Wolf, Klaus Rummler, Patrick Bettinger, und Sandra Aßmann. MedienPädagogik: Zeitschrift für Theorie und Praxis der Medienbildung, Jahrbuch Medienpädagogik, 16 (Jahrbuch Medienpädagogik): 185-209. https://doi.org/10.21240/mpaed/jb16/2021.02.24.X.

Koppel, Ilka. 2021. «Gelingensbedingungen für den Einsatz digitaler Medien: Eine qualitative Studie in der Alphabetisierung und Grundbildung». Herausgegeben von Karsten D. Wolf, Klaus Rummler, Patrick Bettinger, und Sandra Aßmann. MedienPädagogik: Zeitschrift für Theorie und Praxis der Medienbildung, Jahrbuch Medienpädagogik, 16 (Jahrbuch Medienpädagogik): 51-78. https:// doi.org/10.21240/mpaed/jb16/2021.01.12.X.

Mehlan, Henriette, und Jörg Holten. 2021. «Die Dualität von Kompetenz in tiefgreifend mediatisierten Welten: Zum Verständnis eines theoretischen Schlüsselkonzepts der Medienpädagogik». Herausgegeben von Karsten D. Wolf, Klaus Rummler, Patrick Bettinger, und Sandra Aßmann. MedienPädagogik: Zeitschrift für Theorie und Praxis der Medienbildung, Jahrbuch Medienpädagogik, 16 (Jahrbuch Medienpädagogik): 29-49. https://doi.org/10.21240/mpaed/ jb16/2021.01.11.X.

Nickel, Stefanie. 2021. «Räume erschliessen mit einem Smartphone im Sachunterricht an einer Grundschule: Möglichkeiten und Herausforderungen». Herausgegeben von Karsten D. Wolf, Klaus Rummler, Patrick Bettinger, und Sandra Aßmann. MedienPädagogik: Zeitschrift für Theorie und Praxis der Medienbildung, Jahrbuch Medienpädagogik, 16 (Jahrbuch Medienpädagogik): 161-84. https://doi.org/10.21240/mpaed/jb16/2021.01.16.X.

Spanhel, Dieter. 2021. «Aufwachsen in mediatisierten Lebenswelten: Zur Notwendigkeit des Zusammenwirkens von Medienpädagogik und Medienschutz». Herausgegeben von Karsten D. Wolf, Klaus Rummler, Patrick Bettinger, und Sandra Aßmann. MedienPädagogik: Zeitschrift für Theorie und Praxis der Medienbildung, Jahrbuch Medienpädagogik, 16 (Jahrbuch Medienpädagogik): 23180. https://doi.org/10.21240/mpaed/jb16/2021.02.26.X.

Wachs, Sebastian, und Michele F. Wright. 2021. «Associations between Online Hate Victimization and Perpetration: The Buffering Effects of Technical and Assertive Coping». Herausgegeben von Karsten D. Wolf, Klaus Rummler, Patrick Bettinger, und Sandra Aßmann. MedienPädagogik: Zeitschrift für Theorie und Praxis der Medienbildung, Jahrbuch Medienpädagogik, 16 (Jahrbuch Medienpädagogik): 109-28. https://doi.org/10.21240/mpaed/jb16/2021.01.14.X. 